\title{
Heterosis Studies for Fruit Yield and its Components in Tomato (Solanum lycopersicum L.)
}

\author{
N.C. Ghadage*, G.U. Kulkarni, H.R. Meta and Raju Shyadambi
}

Department of Genetics and Plant Breeding, College of Agriculture, Junagadh Agricultural University, Junagadh- 362001 Gujarat (India)

*Corresponding author

\begin{tabular}{|c|c|}
\hline & A B S T R A C T \\
\hline & \multirow{5}{*}{$\begin{array}{l}\text { The experiment was carried out at Vegetable Research Station, Junagadh Agricultural } \\
\text { University, Junagadh, Gujarat during rabi season of } 2017-19 \text { to study performance of nine } \\
\text { lines and four testers into thirty-six tomato hybrids for fruit yield and its component traits } \\
\text { by Line x Tester analysis. The analysis of variance for seventeen characters showed } \\
\text { significant differences for all the genotypes indicating that experimental material had } \\
\text { sufficient genetic variability for all the characters studied. Among } 36 \text { hybrids, } 9 \text { and } 12 \\
\text { hybrids manifested significant desirable heterobeltiosis and standard heterosis for fruit } \\
\text { yield per plant. The heterobeltiosis for fruit yield ranged from }-70.26 \text { to } 206.04 \% \text { while } \\
\text { standard heterosis ranged from }-66.32 \text { to } 189.32 \% \text {. The cross JTL-15-02 } \times \text { Punjab } \\
\text { Chhuhara exhibited the highest desirable heterosis over better parent followed by JTL-15- } \\
05 \times \text { DVRT- } 2 \text {. These hybrids exhibited desirable heterosis for important yield attributes } \\
\text { suggesting that the heterosis for marketable yield was associated with heterosis for } \\
\text { component characters. }\end{array}$} \\
\hline & \\
\hline $\begin{array}{l}\text { Heterobeltiosis, } \\
\text { Standard heterosis, } \\
\text { Component traits } \\
\text { and tomato }\end{array}$ & \\
\hline Article Info & \\
\hline $\begin{array}{l}\text { Accepted: } \\
\text { 10 June } 2019 \\
\text { Available Onlin } \\
10 \text { July } 2019\end{array}$ & \\
\hline
\end{tabular}

\section{Introduction}

Tomato (Solanum lycopersicum L.) is one of the most important world's largest vegetable crop ranks third after potato and onion. It is originated in Peru of South America region (Soni and Soni, 2010).Tomato is mainly grown as rabi crop in the plains of India including tropical, sub-tropical and temperate regions. However, in the hilly region it can also be grown as a summer and rainy season crop. It is a typical day neutral plant and selfpollinated crop but up to $5 \%$ cross pollination also occurs through insects such as bees
(Singh et al., 2004). It is a warm season crop reasonably resistant to heat, drought and grows under wide range of soil and climatic conditions. Optimum temperature for tomato cultivation is $20-24^{\circ} \mathrm{C}$. The annual worldwide production of tomatoes has been estimated at 177.8 million tonnes from total production area of about 4.7 Mha with a productivity of 37.01 tonnes/ha (FAO, 2016). India ranks $2^{\text {nd }}$ in the world with the total area of 0.80 Mha with production and productivity of 19759.92 tonnes and 25 tonnes/ha, respectively (Indian Horticulture Database, 2018). 
In tomato, great amount of heterosis including standard heterosis has been reported by many research workers. Hedrick and Booth (1908) were the first research workers to realize the phenomenon of hybrid vigour in tomato. Heterosis manifestation in tomato is in the form of the greater vigour, faster growth and development, earliness in maturity, increased productivity (Yordanov, 1983). So a speedy improvement can be brought about by exploiting heterosis for fruit yield and its component traits.

\section{Materials and Methods}

The present investigation was conducted at Vegetable Research Station, Junagadh Agricultural University, Junagadh, during late kharif2017-18 for development of crosses and late kharif2018-19 for evaluation of crosses and parents. Geographically, Junagadh is situated at $21.5^{\circ} \mathrm{N}$ latitude and $70.5^{\circ} \mathrm{E}$ longitude with an altitude of 60 meters above the mean sea level. Experimental material contains nine lines (females) namely, JTL-1505, JTL-12-07, JTL-16-03, JTL-16-07, JTL15-02, JTL-12-02， JTL-16-05， JTL-16-08, JTL-17-06 and four testers (males) i.e. JT-3, AT-3, DVRT-2, Punjab Chhuharaof tomato (Solanum lycopersicum L.) were selected on the basis of their phenotypic variability resulted into thirty-six crosses along with their thirteen parents including one standard check variety (JT-3) was evaluated. The experiment was laid out in a Randomized Block design (RBD) with three replications. Five competitive plants were randomly selected for recording the observations on different characters such as days to $50 \%$ flowering, plant height $(\mathrm{cm})$, number of branches per plant, number of clusters per plant, number of fruits per cluster, total number of fruits per plant days to first harvest, fruit yield per plant $(\mathrm{kg})$, average fruit weight (g), numbers of locules per fruit, pericarp thickness $(\mathrm{mm})$, total numbers of pickings, fruit polar diameter $(\mathrm{cm})$, fruit equatorial diameter $(\mathrm{cm})$, days to last harvest, total soluble solid ( ${ }^{\mathrm{o}}$ Brix) and acidity (\%).Analysis of variance were done as suggested by Panse and Sukhatme (1967).

\section{Results and Discussion}

There were significant differences among the parental lines with respect to different characters studied including yield per plant. The mean performance of eight parental lines along with $36 \mathrm{~F}_{1}$ hybrids is given in table 1 and 2. Heterobeltiosis for days to $50 \%$ flowering over better parent ranged from 9.60 per cent (JTL-15-02 $\times$ JT-3) to 11.02 per cent (JTL-16-05 $\times$ DVRT-2). Heterosis over standard check ranged from -2.70 per cent (JTL-12-02 $\times$ AT-3) to 18.02 per cent (JTL16-05 $\times$ DVRT-2). Significant negative heterosis also been reported by Droka et al., (2013), Sureshkumara et al., (2017), Adnan et al., (2018) and Kattegoudar et al., (2018). Heterobeltiosis for plant height over better parent ranged from -41.60 per cent (JTL-12$07 \times$ JT-3) to 42.66 per cent $($ JTL-12-07 $\times$ DVRT-2). The heterosis over standard check ranged from -43.78 per cent (JTL-16-08 $\times$ AT-3) to 23.32 per cent (JTL-12-02 $\times$ AT-3). These results are in confirmation with the results of Yadav et al., (2013), Ahmad et al., (2015), Marbhal et al., (2016), Adnan et al., (2018), Gautam et al., (2018), Kattegoudar et al., (2018) and Sundharaiya et al., (2018).

Heterosis over better parent for number of branches per plant ranged from -25.64 per cent (JTL-16-08 $\times$ AT-3) to 28.77 per cent (JTL-16-03 $\times$ JT-3). Heterosis over standard check ranged from -13.43 per cent (JTL-16$08 \times$ AT-3) to 56.72 per cent (JTL-16-07 $\times$ Punjab Chhuhara). These results are in confirmation with the results of Droka et al., (2013), Sureshkumara et al., (2017), Kattegoudar et al., (2018), Sundharaiya et al., (2018).Heterosis over better parent for 
number of clusters per plant ranged from 38.84 per cent (JTL-16-08 $\times$ AT-3) to 57.84 per cent $(\mathrm{JTL}-16-08 \times \mathrm{JT}-3)$. Heterosis over standard check ranged from -27.45 per cent (JTL-16-08 $\times$ AT-3) to 82.35 per cent (JTL15-05 $\times$ JT-3). These results are in confirmation with the results of Sekhar et al., (2010), Marbhal et al., (2016). The range of heterosis over better parent for Number of fruits per cluster was recorded from -30.91 per cent (JTL-17-06 $\times$ DVRT-2) to 46.51 per cent (JTL-16-05 $\times$ Punjab Chhuhara). Heterosis over standard check ranged from 24.00 per cent (JTL-17-06 $\times$ DVRT-2) to 26.00 per cent (JTL-16-05 $\times$ Punjab Chhuhara). Similar findings were reported by Ahmad et al., (2015), Marbhal et al., (2016), Sureshkumara et al., (2017), Adnan et al., (2018).

The magnitude of heterosis over better parent for total number of fruits per plant ranged from -76.56 per cent (JTL-16-03 × Punjab Chhuhara) to 100.00 per cent (JTL-15-05 $\times$ JT-3 and JTL-15-02 $\times$ Punjab Chhuhara). Heterosis over standard check ranged from 64.21 per cent (JTL-16-08 $\times$ AT-3) to 129.47 per cent (JTL-15-05 $\times$ DVRT-2 and JTL-15$05 \times$ JT-3). Similar findings were reported by Yadav et al., (2013), Gautam et al., (2018), Kattegoudar et al., (2018), Sundharaiya et al., (2018).The range of heterosis over better parent for days to first harvest was recorded from -10.75 per cent (JTL-16-05 × Punjab Chhuhara) to 9.71 per cent (JTL-12-07 $\times$ AT$3)$. Heterosis over standard check ranged from -1.32 per cent (JTL-16-05 × Punjab Chhuhara) to 14.85 per cent (JTL-16-05 $\times$ AT-3). Significant negative heterosis for this character has been reported by Rana and Vidyasagar (2005), Rattan (2007), Droka et al., (2013). The range of heterosis over better parent for fruit yield per plant $(\mathrm{Kg})$ recorded from -70.26 per cent (JTL-16-08 × DVRT-2) to 206.04 per cent (JTL-15-02 $\times$ Punjab Chhuhara). The range of standard heterosis for this trait varied from -66.32 per cent (JTL$16-08 \times$ AT-3) to 189.32 per cent (JTL-15-05 $\times$ DVRT-2).Similar findings were reported by Panchal et al., (2017), Sureshkumara et al., (2017), Tamata and Singh (2017), Triveni et al., (2017), Kattegoudar et al., (2018), Sundharaiya et al., (2018).

The range of heterosis over better parent for average fruit weight $(\mathrm{g})$ recorded from -46.12 per cent (JTL-15-05 $\times$ JT-3) to 34.71 per cent (JTL-15-02 $\times$ Punjab Chhuhara). Heterosis over standard check ranged from -35.37 per cent (JTL-16-08 $\times$ Punjab Chhuhara) to 32.87 per cent (JTL-16-05 $\times$ JT-3). Significant positive heterosis for this character has been reported by Sureshkumara et al., (2017), Tamata and Singh (2017), Gautam et al., (2018), Kattegoudar et al., (2018). The range of heterosis over better parent for number of locules per fruit was recorded from -33.33 per cent (JTL-17-06 $\times$ JT-3) to 44.00 per cent $($ JTL-15-02 $\times$ JT-3). Heterosis over standard check ranged from -10.64 per cent (JTL-17$06 \times \mathrm{JT}-3)$ to 53.19 per cent $(\mathrm{JTL}-15-02 \times \mathrm{JT}-$ 3). Significant positive heterosis for this character has been reported by Garg et al., (2013), Sureshkumara et al., (2017), Sharma and Singh (2018). The range of heterosis over better parent for pericarp thickness $(\mathrm{mm})$ was recorded from -22.90 per cent (JTL-15-02 $\times$ Punjab Chhuhara) to 14.68 per cent (JTL-17$06 \times$ DVRT-2). Heterosis over standard check ranged from -10.85 per cent (JTL-15-02 $\times$ Punjab Chhuhara) to 25.33 per cent (JTL-16$05 \times$ Punjab Chhuhara). Significant positive heterosis for this character has been reported by Joshi et al., (2005), Rattan (2007), Garg et al., (2013), Sureshkumara et al., (2017).

Heterobeltiosis for total number of pickings ranged from -45.16 per cent (JTL-16-08 $\times$ DVRT-2) to 15.38 per cent (JTL-16-07 $\times$ JT$3)$. 
Table.1 Mean value for yield and yield attributing traits of the parents.

\begin{tabular}{|c|c|c|c|c|c|c|c|c|c|}
\hline Genotype & $\begin{array}{c}\text { Days to } 50 \% \\
\text { Flowering }\end{array}$ & Plant height (cm) & $\begin{array}{c}\text { Number of } \\
\text { branches per } \\
\text { plant }\end{array}$ & $\begin{array}{l}\text { Number of } \\
\text { clusters per } \\
\text { plant }\end{array}$ & $\begin{array}{l}\text { Number of } \\
\text { fruits per } \\
\text { cluster }\end{array}$ & $\begin{array}{c}\text { Total number } \\
\text { of fruits per } \\
\text { plant }\end{array}$ & $\begin{array}{c}\text { Days to first } \\
\text { harvest }\end{array}$ & $\begin{array}{l}\text { Fruit yield per } \\
\text { plant }(\mathrm{Kg})\end{array}$ & $\begin{array}{c}\text { Average fruit } \\
\text { weight (g) }\end{array}$ \\
\hline \multicolumn{10}{|l|}{ Lines } \\
\hline JTL-15-05 & 39.00 & 116.47 & 6.40 & 10.73 & 3.20 & 36.33 & 107.67 & 3.10 & 71.25 \\
\hline JTL-12-07 & 37.67 & 82.47 & 6.13 & 8.87 & 3.13 & 51.67 & 103.00 & 2.88 & 56.20 \\
\hline JTL-16-03 & 37.33 & 105.67 & 4.87 & 9.60 & 3.27 & 64.00 & 103.67 & 2.69 & 42.98 \\
\hline JTL-16-07 & 40.33 & 107.40 & 6.07 & 10.33 & 3.60 & 42.33 & 106.67 & 1.87 & 43.63 \\
\hline JTL-15-02 & 41.67 & 89.47 & 5.67 & 7.53 & 3.40 & 22.33 & 106.67 & 0.91 & 39.15 \\
\hline JTL-12-02 & 39.00 & 110.53 & 6.07 & 7.13 & 3.13 & 21.00 & 112.67 & 0.62 & 32.97 \\
\hline JTL-16-05 & 39.33 & 107.33 & 5.20 & 7.93 & 2.80 & 24.33 & 111.67 & 1.14 & 44.25 \\
\hline JTL-16-08 & 36.33 & 70.80 & 5.20 & 6.33 & 3.27 & 24.67 & 111.67 & 1.22 & 36.79 \\
\hline JTL-17-06 & 39.33 & 79.73 & 5.20 & 5.93 & 3.67 & 19.00 & 103.67 & 0.91 & 38.39 \\
\hline \multicolumn{10}{|l|}{ Testers } \\
\hline JT-3 (Standard check) & 37.00 & 116.33 & 4.47 & 6.80 & 3.33 & 31.67 & 101.00 & 1.62 & 52.48 \\
\hline AT-3 & 37.00 & 99.20 & 5.20 & 8.07 & 3.53 & 37.00 & 102.33 & 1.35 & 37.13 \\
\hline DVRT-2 & 38.00 & 89.07 & 5.20 & 8.20 & 3.00 & 41.00 & 105.67 & 2.57 & 51.59 \\
\hline Punjab Chhuhara & 38.00 & 114.20 & 3.20 & 4.93 & 2.87 & 34.67 & 104.33 & 1.21 & 34.92 \\
\hline
\end{tabular}

\begin{tabular}{|c|c|c|c|c|c|c|c|c|}
\hline Genotype & $\begin{array}{l}\text { Number of } \\
\text { locules per fruit }\end{array}$ & $\begin{array}{c}\text { Pericarp } \\
\text { thickness (mm) }\end{array}$ & $\begin{array}{l}\text { Total numbers } \\
\text { of picking }\end{array}$ & $\begin{array}{c}\text { Fruit polar } \\
\text { diameter }(\mathbf{c m})\end{array}$ & $\begin{array}{l}\text { Fruit equatorial } \\
\text { diameter }(\mathbf{c m})\end{array}$ & $\begin{array}{l}\text { Days to last } \\
\text { harvest }\end{array}$ & 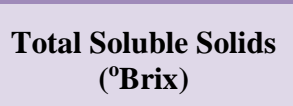 & Acidity \% \\
\hline \multicolumn{9}{|l|}{ Lines } \\
\hline JTL-15-05 & 3.40 & 4.44 & 9.33 & 4.10 & 4.58 & 174.33 & 5.51 & 0.67 \\
\hline JTL-12-07 & 3.80 & 4.80 & 9.67 & 4.08 & 4.28 & 181.33 & 5.31 & 0.70 \\
\hline JTL-16-03 & 3.80 & 4.75 & 9.33 & 3.70 & 3.74 & 179.00 & 4.91 & 0.60 \\
\hline JTL-16-07 & 4.00 & 4.76 & 8.00 & 3.73 & 3.95 & 176.67 & 5.84 & 0.82 \\
\hline JTL-15-02 & 3.33 & 4.19 & 6.33 & 3.87 & 4.24 & 163.67 & 5.29 & 0.56 \\
\hline JTL-12-02 & 3.60 & 4.90 & 6.00 & 3.97 & 4.35 & 160.33 & 4.83 & 0.63 \\
\hline JTL-16-05 & 3.33 & 4.34 & 6.33 & 4.39 & 4.42 & 163.67 & 4.97 & 0.51 \\
\hline JTL-16-08 & 3.00 & 4.92 & 7.00 & 4.36 & 4.64 & 167.33 & 4.36 & 0.73 \\
\hline JTL-17-06 & 4.20 & 4.61 & 4.00 & 4.33 & 4.60 & 159.00 & 5.13 & 0.69 \\
\hline \multicolumn{9}{|l|}{ Testers } \\
\hline JT-3 (Standard check) & 3.13 & 4.33 & 8.67 & 4.12 & 4.64 & 167.33 & 5.00 & 0.69 \\
\hline AT -3 & 3.00 & 4.96 & 9.00 & 4.54 & 4.61 & 172.00 & 5.00 & 1.04 \\
\hline DVRT-2 & 3.27 & 4.56 & 10.33 & 3.97 & 4.03 & 181.33 & 4.57 & 0.68 \\
\hline Punjab Chhuhara & 2.47 & 5.01 & 9.00 & 6.22 & 3.45 & 167.33 & 5.79 & 0.80 \\
\hline
\end{tabular}


Table.2 Mean value for yield and yield attributing traits of the $F_{1}$ hybrids

\begin{tabular}{|c|c|c|c|c|c|c|c|c|c|c|c|c|c|c|c|}
\hline \multirow[t]{2}{*}{ Genotype } & \multicolumn{3}{|c|}{ Days to $50 \%$ Flowering } & \multicolumn{3}{|c|}{ Plant height $(\mathbf{c m})$} & \multicolumn{3}{|c|}{ Number of branches per plant } & \multicolumn{3}{|c|}{ Number of clusters per plant } & \multicolumn{3}{|c|}{ Number of fruits per cluste } \\
\hline & Mean & $\mathrm{H}_{1}(\%)$ & $\mathrm{H}_{2}(\%)$ & Mean & $\mathrm{H}_{1}(\%)$ & $\mathrm{H}_{2}(\%)$ & Mean & $\mathrm{H}_{1}(\%)$ & $\mathrm{H}_{2}(\%)$ & Mean & $\mathrm{H}_{1}(\%)$ & $\mathrm{H}_{2}(\%)$ & Mean & $\mathrm{H}_{1}(\%)$ & $\mathrm{H}_{2}(\%)$ \\
\hline JTL-15-05 $\times$ JT-3 & 37.33 & -4.27 & 0.90 & 80.67 & $-30.74 *$ & $-30.66 *$ & 6.13 & -4.17 & $37.31 *$ & 12.40 & 15.53 & $82.35 * *$ & 3.47 & 4.00 & 4.00 \\
\hline JTL-15-05 $\times$ AT-3 & 37.67 & -3.42 & 1.80 & 101.80 & -12.59 & -12.49 & 5.60 & -12.50 & 25.37 & 11.20 & 4.35 & $64.71 * *$ & 3.33 & -5.66 & 0.00 \\
\hline JTL-15-05 $\times$ DVRT-2 & 38.67 & -0.85 & 4.50 & 104.60 & -10.19 & -10.09 & 5.80 & -9.38 & 29.85 & 11.73 & 9.32 & $72.55 * *$ & 3.83 & 19.79 & 15.00 \\
\hline JTL-15-05 $\times$ Punjab Chhuhara & 37.67 & -3.42 & 1.80 & 69.00 & $-40.76 * *$ & $-40.69 * *$ & 6.27 & -2.08 & $40.30 *$ & 12.13 & 13.04 & $78.43 * *$ & 3.80 & 18.75 & 14.00 \\
\hline JTL-12-07 $\times$ JT-3 & 38.67 & 2.65 & 4.50 & 67.93 & $-41.60 * *$ & $-41.60 * *$ & 5.27 & -14.13 & 17.91 & 11.60 & 30.83 & 70.59 ** & 3.27 & -2.00 & -2.00 \\
\hline JTL-12-07 $\times$ AT-3 & 40.00 & 6.19 & 8.11 & 119.70 & 20.67 & 2.89 & 6.80 & 10.87 & $52.24 * *$ & 10.80 & 21.80 & $58.82 * *$ & 3.53 & 0.00 & 6.00 \\
\hline JTL-12-07 $\times$ DVRT-2 & 37.33 & -1.75 & 0.90 & 127.07 & $42.66 *$ & 9.23 & 6.87 & 11.96 & 53.73 ** & 11.33 & 27.82 & $66.67 * *$ & 3.13 & 0.00 & -6.00 \\
\hline JTL-12-07 $\times$ Punjab Chhuhara & 38.33 & 0.88 & 3.60 & 114.40 & 0.18 & -1.66 & 6.33 & 3.26 & $41.79 *$ & 6.00 & $-32.33 *$ & -11.76 & 4.00 & $27.66 *$ & 20.00 \\
\hline JTL-16-03 $\times$ JT-3 & 39.33 & 5.36 & 6.31 & 122.73 & 5.50 & 5.50 & 6.27 & 28.77 & $40.30 *$ & 11.47 & 19.44 & 68.63 ** & 3.13 & -6.00 & -6.00 \\
\hline JTL-16-03 $\times$ AT-3 & 41.00 & $9.82 *$ & 10.81 & 113.67 & 7.57 & -2.29 & 6.00 & 15.38 & $34.33 *$ & 6.47 & $-32.64 *$ & -4.90 & 3.33 & -5.66 & 0.00 \\
\hline JTL-16-03 × DVRT-2 & 37.67 & -0.88 & 1.80 & 120.40 & 13.94 & 3.50 & 5.80 & 11.54 & 29.85 & 8.93 & -6.94 & 31.37 & 3.17 & -3.06 & -5.00 \\
\hline JTL-16-03 $\times$ Punjab Chhuhara & 41.00 & 7.89 & 10.81 & 133.20 & 16.64 & 14.50 & 5.67 & 16.44 & 26.87 & 6.93 & -27.78 & 1.96 & 2.67 & -18.37 & -20.00 \\
\hline JTL-16-07 $\times$ JT-3 & 39.33 & -2.48 & 6.31 & 114.47 & -1.60 & -1.60 & 6.80 & 12.09 & $52.24 * *$ & 7.87 & -23.87 & 15.69 & 3.53 & -1.85 & 6.00 \\
\hline JTL-16-07 $\times$ AT-3 & 39.33 & -2.48 & 6.31 & 110.00 & 2.42 & -5.44 & 5.93 & -2.20 & $32.84 *$ & 10.13 & -1.94 & $49.02 *$ & 3.10 & -13.89 & -7.00 \\
\hline JTL-16-07 $\times$ DVRT-2 & 40.67 & 0.83 & 9.91 & 123.60 & 15.08 & 6.25 & 5.93 & -2.20 & $32.84 *$ & 9.80 & -5.16 & $44.12 *$ & 3.53 & -1.85 & 6.00 \\
\hline JTL-16-07 $\times$ Punjab Chhuhara & 37.33 & -7.44 & 0.90 & 122.73 & 7.47 & 5.50 & 7.00 & 15.38 & $56.72 * *$ & 9.73 & -5.81 & $43.14 *$ & 3.40 & -5.56 & 2.00 \\
\hline JTL-15-02 $\times$ JT-3 & 37.67 & $-9.60^{*}$ & 1.80 & 95.20 & -18.17 & -18.17 & 6.40 & 12.94 & $43.28 *$ & 7.60 & 0.88 & 11.76 & 3.13 & -7.84 & -6.00 \\
\hline JTL-15-02 $\times$ AT-3 & 39.00 & -6.40 & 5.41 & 89.20 & -10.08 & -23.32 & 5.53 & -2.35 & 23.88 & 9.53 & 18.18 & 40.20 & 3.40 & -3.77 & 2.00 \\
\hline JTL-15-02 $\times$ DVRT-2 & 38.67 & -7.20 & 4.50 & 95.13 & 6.33 & -18.22 & 5.60 & -1.18 & 25.37 & 10.80 & 31.71 & $58.82 * *$ & 3.47 & 1.96 & 4.00 \\
\hline JTL-15-02 $\times$ Punjab Chhuhara & 40.33 & -3.20 & 9.01 & 94.87 & -16.93 & -18.45 & 5.13 & -9.41 & 14.93 & 11.87 & $57.52 * *$ & $74.51 * *$ & 3.83 & 12.75 & 15.00 \\
\hline JTL-12-02 $\times$ JT-3 & 40.67 & 4.27 & 9.91 & 115.47 & -0.74 & -0.74 & 5.67 & -6.59 & 26.87 & 9.67 & 35.51 & $42.16 *$ & 3.13 & -6.00 & -6.00 \\
\hline JTL-12-02 $\times$ AT-3 & 36.00 & -7.69 & -2.70 & 143.47 & $29.79 *$ & 23.32 & 6.13 & 1.10 & $37.31 *$ & 7.27 & -9.92 & 6.86 & 3.90 & 10.38 & 17.00 \\
\hline JTL-12-02 $\times$ DVRT-2 & 39.00 & 0.00 & 5.41 & 118.27 & 7.00 & 1.66 & 5.87 & -3.30 & 31.34 & 10.40 & 26.83 & $52.94 *$ & 3.00 & -4.26 & -10.00 \\
\hline JTL-12-02 $\times$ Punjab Chhuhara & 37.67 & -3.42 & 1.80 & 139.33 & 22.01 & 19.77 & 6.20 & 2.20 & $38.81 *$ & 7.27 & 1.87 & 6.86 & 3.40 & 8.51 & 2.00 \\
\hline JTL-16-05 $\times$ JT-3 & 40.33 & 2.54 & 9.01 & 126.20 & 8.48 & 8.48 & 5.60 & 7.69 & 25.37 & 10.87 & $36.97 *$ & $59.80 * *$ & 3.27 & -2.00 & -2.00 \\
\hline JTL-16-05 × AT-3 & 41.00 & 4.24 & 10.81 & 129.13 & 20.31 & 11.00 & 6.67 & $28.21 *$ & $49.25 * *$ & 11.13 & $38.02 *$ & $63.73 * *$ & 3.27 & -7.55 & -2.00 \\
\hline JTL-16-05 × DVRT-2 & 43.67 & $11.02 * *$ & 18.02 & 117.73 & 9.69 & 1.20 & 6.27 & 20.51 & $40.30 *$ & 8.47 & 3.25 & 24.51 & 3.30 & 10.00 & -1.00 \\
\hline JTL-16-05 $\times$ Punjab Chhuhara & 36.67 & -6.78 & -0.90 & 102.53 & -10.22 & -11.86 & 6.60 & 26.92 & $47.76 * *$ & 9.87 & 24.37 & $45.10 *$ & 4.20 & $46.51 * *$ & $26.00 *$ \\
\hline JTL-16-08 $\times$ JT-3 & 37.33 & 0.90 & 0.90 & 74.73 & $-35.76 * *$ & $-35.76 * *$ & 5.67 & 8.97 & 26.87 & 10.73 & $57.84 * *$ & $57.84 * *$ & 3.53 & 6.00 & 6.00 \\
\hline JTL-16-08 $\times$ AT-3 & 39.67 & 7.21 & 7.21 & 65.40 & $-34.07 *$ & $-43.78 * *$ & 3.87 & -25.64 & -13.43 & 4.93 & $-38.84 *$ & -27.45 & 3.53 & 0.00 & 6.00 \\
\hline JTL-16-08 $\times$ DVRT-2 & 41.67 & $9.65^{*}$ & 12.61 & 92.33 & 3.67 & -20.63 & 4.93 & -5.13 & 10.45 & 9.07 & 10.57 & 33.33 & 3.00 & -8.16 & -10.00 \\
\hline JTL-16-08 $\times$ Punjab Chhuhara & 37.00 & -2.63 & 0.00 & 83.80 & -26.62 & $-27.97 *$ & 5.13 & -1.28 & 14.93 & 9.27 & $46.32 *$ & 36.27 & 3.07 & -6.12 & -8.00 \\
\hline JTL-17-06 $\times$ JT-3 & 38.00 & -3.39 & 2.70 & 119.20 & 2.46 & 2.46 & 5.00 & -3.85 & 11.94 & 9.07 & 33.33 & 33.33 & 3.80 & 3.64 & 14.00 \\
\hline JTL-17-06 $\times$ AT-3 & 37.33 & -5.08 & 0.90 & 124.40 & 25.40 & 6.93 & 5.73 & 10.26 & 28.36 & 9.67 & 19.83 & $42.16 *$ & 3.13 & -14.55 & -6.00 \\
\hline JTL-17-06 $\times$ DVRT-2 & 41.00 & 4.24 & 10.81 & 117.13 & 31.51 & 0.69 & 4.80 & -7.69 & 7.46 & 10.07 & 22.76 & $48.04 *$ & 2.53 & $-30.91 * *$ & $-24.00 *$ \\
\hline JTL-17-06 $\times$ Punjab Chhuhara & 38.33 & -2.54 & 3.60 & 108.27 & -5.20 & -6.93 & 5.50 & 5.77 & 23.13 & 8.13 & 37.08 & 19.61 & 3.27 & -10.91 & -2.00 \\
\hline Mean & 38.82 & & & 105.84 & & & 5.70 & & & 9.11 & & & 3.33 & & \\
\hline C.D $(5 \%)$ & 3.15 & & & 30.69 & & & 1.45 & & & 2.77 & & & 0.68 & & \\
\hline C.V \% & 5.01 & & & 17.87 & & & 15.71 & & & 18.76 & & & 12.7 & & \\
\hline
\end{tabular}


Table.2 (Contd.) Mean value for yield and yield attributing traits of the $\mathrm{F}_{1}$ hybrids

\begin{tabular}{|c|c|c|c|c|c|c|c|c|c|c|c|c|c|c|c|c|c|c|}
\hline \multirow[t]{2}{*}{ Genotype } & \multicolumn{3}{|c|}{ Total no. of fruits per plant } & \multicolumn{3}{|c|}{ Days to first harvest } & \multicolumn{3}{|c|}{ Fruit yield per plant (Kg) } & \multicolumn{3}{|c|}{ Average fruit weight (g) } & \multicolumn{3}{|c|}{ Number of locules per fruit } & \multicolumn{3}{|c|}{ Pericarp thickness (mm) } \\
\hline & Mean & $\mathrm{H}_{1}(\%)$ & $\mathrm{H}_{2}(\%)$ & Mean & $\mathrm{H}_{1}(\%)$ & $\mathrm{H}_{2}(\%)$ & Mean & $\mathrm{H}_{1}(\%)$ & $\mathrm{H}_{2}(\%)$ & Mean & $\mathrm{H}_{1}(\%)$ & $\mathrm{H}_{2}(\%)$ & Mean & $\mathrm{H}_{1}(\%)$ & $\mathrm{H}_{2}(\%)$ & Mean & $\mathrm{H}_{1}(\%)$ & $\mathrm{H}_{2}(\%)$ \\
\hline JTL-15-05 × JT-3 & 72.67 & $100.00^{* *}$ & $129.47 * *$ & 107.33 & -0.31 & 6.27 & 2.87 & -7.43 & $76.59 * *$ & 38.39 & $-46.12 * *$ & $-26.85^{*}$ & 3.07 & -9.80 & -2.13 & 4.94 & 11.41 & 14.24 \\
\hline JTL-15-05 × AT-3 & 71.00 & $91.89 * *$ & $124.21 * *$ & 107.00 & -0.62 & 5.94 & 2.93 & -5.38 & 80.49 ** & 46.38 & $-34.90 * *$ & -11.62 & 3.33 & -1.96 & 6.38 & 4.75 & -4.10 & 9.78 \\
\hline JTL-15-05 × DVRT-2 & 72.67 & $77.24 * *$ & $129.47 * *$ & 106.33 & -1.24 & 5.28 & 4.70 & $51.67 * *$ & $189.32 * *$ & 64.77 & -9.09 & 23.43 & 3.20 & -5.88 & 2.13 & 4.86 & 6.73 & 12.32 \\
\hline JTL-15-05 × Punjab Chhuhara & 18.00 & $-50.46 * *$ & $-43.16^{*}$ & 110.00 & 2.17 & $8.91 *$ & 1.42 & $-54.14 * *$ & -12.53 & 57.75 & $-18.94 *$ & 10.06 & 3.60 & 5.88 & 14.89 & 4.59 & -8.32 & 6.00 \\
\hline JTL-12-07 $\times$ JT-3 & 50.67 & -1.94 & $60.00 * *$ & 108.67 & 5.50 & $7.59 *$ & 3.09 & 7.28 & 90.55 ** & 57.72 & 2.70 & 9.99 & 3.20 & -15.79 & 2.13 & 4.80 & 0.14 & 10.85 \\
\hline JTL-12-07 × AT-3 & 52.33 & 1.29 & $65.26 * *$ & 113.00 & $9.71 * *$ & $11.88 * *$ & 2.34 & -18.96 & $43.94 *$ & 51.61 & -8.17 & -1.65 & 3.60 & -5.26 & 14.89 & 4.49 & -9.48 & 3.62 \\
\hline JTL-12-07 × DVRT-2 & 42.33 & -18.06 & 33.68 & 109.00 & 3.15 & $7.92 *$ & 2.32 & -19.77 & $42.51 *$ & 53.39 & -5.00 & 1.74 & 3.40 & -10.53 & 8.51 & 5.20 & 8.48 & $20.09 *$ \\
\hline JTL-12-07 × Punjab Chhuhara & 14.00 & $-72.90 * *$ & $-55.79 * *$ & 107.33 & 2.88 & 6.27 & 1.03 & $-64.28 * *$ & -36.55 & 60.26 & 7.22 & 14.83 & 3.53 & -7.02 & 12.77 & 4.73 & -5.59 & 9.16 \\
\hline JTL-16-03 $\times$ JT-3 & 33.33 & $-47.92 * *$ & 5.26 & 105.67 & 1.93 & 4.62 & 2.17 & -19.23 & 33.68 & 47.99 & -8.56 & -8.56 & 3.47 & -8.77 & 10.64 & 4.85 & 2.18 & 12.01 \\
\hline JTL-16-03 × AT-3 & 26.33 & $-58.85 * *$ & -16.84 & 110.00 & 6.11 & $8.91 *$ & 1.42 & $-47.27 * *$ & -12.73 & 47.43 & 10.36 & -9.61 & 3.53 & -7.02 & 12.77 & 4.24 & -14.39 & -2.00 \\
\hline JTL-16-03 × DVRT-2 & 33.00 & $-48.44 * *$ & 4.21 & 107.33 & 1.58 & 6.27 & 2.30 & -14.39 & $41.68 *$ & 55.07 & 6.75 & 4.94 & 3.07 & -19.30 & -2.13 & 4.41 & -7.02 & 1.92 \\
\hline JTL-16-03 $\times$ Punjab Chhuhara & 15.00 & $-76.56 * *$ & $-52.63 * *$ & 111.33 & 6.71 & $10.23 * *$ & 0.93 & $-65.38 * *$ & $-42.71 *$ & 42.27 & -1.66 & -19.46 & 2.87 & $-24.56 *$ & -8.51 & 4.71 & -5.99 & 8.70 \\
\hline JTL-16-07 $\times$ JT-3 & 42.00 & -0.79 & 32.63 & 103.00 & -3.44 & 1.98 & 1.73 & -7.3 & 6.98 & 38.87 & $-25.94 *$ & $-25.94 *$ & 3.27 & -18.33 & 4.26 & 4.44 & -6.86 & 2.39 \\
\hline JTL-16-07 $\times$ AT-3 & 67.67 & $59.84 * *$ & $113.68 * *$ & 104.00 & -2.50 & 2.97 & 3.12 & $66.73 * *$ & $92.40 * *$ & 44.34 & 1.63 & -15.50 & 3.47 & -13.33 & 10.64 & 4.77 & -3.70 & 10.24 \\
\hline JTL-16-07 × DVRT-2 & 33.00 & -22.05 & 4.21 & 105.00 & -1.56 & 3.96 & 1.93 & $-24.68 *$ & 19.10 & 50.49 & -2.13 & -3.79 & 4.00 & 0.00 & $27.66 *$ & 4.27 & -10.36 & -1.46 \\
\hline JTL-16-07 × Punjab Chhuhara & 24.67 & $-41.73 * *$ & -22.11 & 107.67 & 0.94 & 6.60 & 1.42 & -24.38 & -12.73 & 49.13 & 12.61 & & 3.73 & -6.67 & 19.15 & 4.22 & $-15.71 *$ & -2.54 \\
\hline JTL-15-02 $\times$ JT-3 & 50.67 & $60.00 * *$ & $60.00 * *$ & 102.67 & -3.75 & 1.65 & 2.73 & $68.17 * *$ & $68.17 * *$ & 48.41 & -7.74 & -7.74 & 4.80 & $44.00 * *$ & $53.19 * *$ & 3.67 & -15.32 & -15.32 \\
\hline JTL-15-02 $\times$ AT-3 & 40.67 & 9.91 & 28.42 & 104.33 & -2.19 & 3.30 & 1.99 & $47.77 *$ & 22.59 & 44.49 & 13.66 & -15.21 & 2.93 & -12.00 & -6.38 & 4.89 & -1.41 & 12.86 \\
\hline JTL-15-02 $\times$ DVRT-2 & 64.00 & $56.10 * *$ & 102.11 ** & 104.33 & -2.19 & 3.30 & 3.54 & $37.92 * *$ & $118.07 * *$ & 52.21 & 1.20 & -0.51 & 3.20 & -4.00 & 2.13 & 5.23 & 14.78 & $20.79 *$ \\
\hline JTL-15-02 × Punjab Chhuhara & 69.33 & $100.00 * *$ & $118.95 * *$ & 103.33 & -3.13 & 2.31 & 3.72 & $206.04 * *$ & $128.75 * *$ & 52.73 & $34.71 *$ & 0.49 & 3.13 & -6.00 & 0.00 & 3.86 & $-22.90 * *$ & -10.85 \\
\hline JTL-12-02 $\times$ JT-3 & 36.67 & 15.79 & 15.79 & 107.33 & -4.73 & 6.27 & 1.94 & 19.51 & 19.51 & 53.66 & 2.26 & 2.26 & 4.20 & 16.67 & $34.04 *$ & 4.25 & -13.14 & -1.77 \\
\hline JTL-12-02 $\times$ AT-3 & 29.67 & -19.82 & -6.32 & 112.67 & 0.00 & $11.55 * *$ & 1.73 & 28.71 & 6.78 & 48.05 & 29.41 & -8.44 & 3.27 & -9.26 & 4.26 & 4.39 & -11.57 & 1.23 \\
\hline JTL-12-02 $\times$ DVRT-2 & 42.67 & 4.07 & 34.74 & 110.33 & -2.07 & $9.24 *$ & 1.85 & $-27.66 *$ & 14.37 & 43.84 & -15.02 & -16.46 & 3.47 & -3.70 & 10.64 & 4.09 & $-16.47 *$ & -5.54 \\
\hline JTL-12-02 $\times$ Punjab Chhuhara & 30.33 & -12.50 & -4.21 & 109.33 & -2.96 & $8.25 *$ & 1.44 & 18.13 & -11.70 & 45.98 & 31.68 & -12.38 & 3.93 & 9.26 & 25.53 & 4.35 & -13.12 & 0.46 \\
\hline JTL-16-05 × JT-3 & 44.67 & $41.05 *$ & $41.05 *$ & 109.00 & -2.39 & $7.92 *$ & 3.42 & $110.68 * *$ & 110.68 ** & 69.72 & 32.87 ** & $32.87 * *$ & 4.13 & 24.00 & $31.91 *$ & 4.36 & 0.61 & 0.77 \\
\hline JTL-16-05 × AT-3 & 43.33 & 17.12 & 36.84 & 116.00 & 3.88 & $14.85 * *$ & 2.17 & $60.89 * *$ & 33.47 & 48.66 & 9.97 & -7.27 & 3.73 & 12.00 & 19.15 & 4.11 & $-17.01 *$ & -5.00 \\
\hline JTL-16-05 × DVRT-2 & 46.67 & 13.82 & $47.37 *$ & 109.33 & -2.09 & $8.25^{*}$ & 2.17 & -15.32 & 33.88 & 44.65 & -13.45 & -14.92 & 2.93 & -12.00 & -6.38 & 4.66 & 2.27 & 7.62 \\
\hline JTL-16-05 × Punjab Chhuhara & 45.33 & 30.77 & $43.16 *$ & 99.67 & $-10.75^{* *}$ & -1.32 & 1.54 & 26.65 & -5.34 & 33.97 & -23.23 & $-35.27 * *$ & 3.73 & 12.00 & 19.15 & 5.43 & 8.39 & $25.33^{* *}$ \\
\hline JTL-16-08 $\times$ JT-3 & 53.33 & $68.42 * *$ & $68.42 * *$ & 105.00 & -5.97 & 3.96 & 2.19 & 35.11 & 35.11 & 41.33 & -21.25 & -21.25 & 3.07 & -2.13 & -2.13 & 4.85 & -1.36 & 12.01 \\
\hline JTL-16-08 $\times$ AT-3 & 11.33 & $-69.37 * *$ & $-64.21 * *$ & 108.00 & -3.28 & 6.93 & 0.55 & $-59.41 *$ & $-66.32 * *$ & 39.91 & 7.48 & -23.95 & 3.67 & 22.22 & 17.02 & 4.63 & -6.66 & 6.85 \\
\hline JTL-16-08 $\times$ DVRT-2 & 16.67 & $-59.35 * *$ & $-47.37 *$ & 115.67 & 3.58 & $14.52^{* *}$ & 0.76 & $-70.26 * *$ & $-52.98 * *$ & 47.40 & -8.12 & -9.67 & 3.60 & 10.20 & 14.89 & 4.34 & -11.73 & 0.23 \\
\hline JTL-16-08 × Punjab Chhuhara & 14.00 & $-59.62 * *$ & $-55.79 * *$ & 110.67 & -0.90 & $9.57^{* *}$ & 0.62 & -49.32 & $-61.81 * *$ & 33.91 & -7.82 & $-35.37 * *$ & 3.20 & 6.67 & 2.13 & 4.41 & -11.98 & 1.77 \\
\hline JTL-17-06 $\times$ JT-3 & 33.67 & 6.32 & 6.32 & 110.00 & 6.11 & $8.91^{*}$ & 2.05 & 26.28 & 26.28 & 47.67 & -9.17 & -9.17 & 2.80 & $-33.33 * *$ & -10.64 & 4.50 & -2.46 & 3.85 \\
\hline JTL-17-06 × AT-3 & 29.33 & -20.72 & -7.37 & 108.00 & 4.18 & 6.93 & 1.44 & 6.93 & -11.29 & 43.93 & 14.43 & -16.29 & 3.67 & -12.70 & 17.02 & 4.41 & -10.96 & 1.92 \\
\hline JTL-17-06 × DVRT-2 & 41.33 & 0.81 & 30.53 & 108.00 & 2.21 & 6.93 & 2.17 & -15.32 & 33.88 & 48.39 & -6.19 & -7.78 & 2.93 & $-30.16 * *$ & -6.38 & 5.29 & 14.68 & $22.09 *$ \\
\hline JTL-17-06 $\times$ Punjab Chhuhara & 42.67 & 23.08 & 34.74 & 109.67 & 5.11 & $8.58^{*}$ & 1.90 & $56.32 *$ & 16.84 & 44.02 & 14.67 & -16.12 & 3.60 & -14.29 & 14.89 & 4.91 & -1.93 & 13.39 \\
\hline Mean & 38.87 & & & 107.48 & & & 1.99 & & & 47.35 & & & 3.44 & & & 4.60 & & \\
\hline C.D (5\%) & \multicolumn{3}{|l|}{12.54} & \multicolumn{3}{|l|}{7.44} & \multicolumn{3}{|l|}{0.63} & \multicolumn{3}{|l|}{14.86} & \multicolumn{3}{|l|}{0.78} & \multicolumn{3}{|l|}{0.75} \\
\hline C.V \% & 19.88 & & & 4.26 & & & 19.63 & & & 19.34 & & & 14.09 & & & 10.1 & & \\
\hline
\end{tabular}


Table.2 (Contd.) Mean value for yield and yield attributing traits of the $\mathrm{F}_{1}$ hybrids

\begin{tabular}{|c|c|c|c|c|c|c|c|c|c|c|c|c|c|c|c|c|c|c|}
\hline \multirow[t]{2}{*}{ Genotype } & \multicolumn{3}{|c|}{ Total numbers of picking } & \multicolumn{3}{|c|}{ Fruit polar diameter $(\mathbf{c m})$} & \multicolumn{3}{|c|}{$\begin{array}{l}\text { Fruit equatorial diameter } \\
\text { (cm) }\end{array}$} & \multicolumn{3}{|c|}{ Days to last harvest } & \multicolumn{3}{|c|}{ Total Soluble Solids ( ${ }^{\circ}$ Brix) } & \multicolumn{3}{|c|}{ Acidity \% } \\
\hline & Mean & $\mathrm{H}_{1}(\%)$ & $\mathrm{H}_{2}(\%)$ & Mean & $\mathrm{H}_{1}(\%)$ & $\mathrm{H}_{2}(\%)$ & Mean & $\mathrm{H}_{1}(\%)$ & $\mathrm{H}_{2}(\%)$ & Mean & $\mathrm{H}_{1}(\%)$ & $\mathrm{H}_{2}(\%)$ & Mean & $\mathrm{H}_{1}(\%)$ & $\mathrm{H}_{2}(\%)$ & Mean & $\mathrm{H}_{1}(\%)$ & $\mathrm{H}_{2}(\%)$ \\
\hline JTL-15-05 $\times$ JT-3 & 10.67 & 14.29 & 23.08 & 4.06 & -1.46 & -1.46 & 4.14 & -10.78 & -10.78 & 186.00 & 6.69 & 11.16 & 5.64 & 2.42 & 12.80 & 0.75 & 8.17 & 8.17 \\
\hline ITL-15-05 × AT-3 & 9.33 & 0.00 & 7.69 & 4.50 & -1.03 & 9.05 & 4.94 & 7.38 & 6.69 & 178.00 & 2.10 & 6.37 & 5.32 & -3.33 & 6.47 & 0.78 & $-25.00 * *$ & 12.50 \\
\hline ITL-15-05 × DVRT-2 & 10.33 & 0.00 & 19.23 & 4.47 & 9.02 & 8.41 & 4.88 & 6.48 & 5.18 & 183.67 & 1.29 & 9.76 & 4.89 & -11.26 & -2.27 & 0.65 & -4.39 & -5.77 \\
\hline ITL-15-05 × Punjab Chhuhara & 6.67 & $-28.57 *$ & -23.08 & 4.18 & $-32.74 * *$ & 1.46 & 4.67 & 2.04 & 0.79 & 147.33 & $-15.49 *$ & -11.95 & 5.18 & -10.59 & 3.60 & 0.90 & 11.20 & $28.85 *$ \\
\hline TTL-12-07 $\times$ JT-3 & 8.67 & -10.34 & 0.00 & 4.14 & 0.40 & 0.40 & 4.65 & 0.29 & 0.29 & 174.67 & -3.68 & 4.38 & 4.99 & -6.02 & -0.13 & 0.69 & -1.44 & -0.96 \\
\hline ITL-12-07 $\times$ AT-3 & 7.00 & $-27.59 *$ & -19.23 & 3.99 & $-12.25 *$ & -3.31 & 4.67 & 1.37 & 0.72 & 183.67 & 1.29 & 9.76 & 5.29 & -0.38 & 5.87 & 0.66 & $-36.54 * *$ & -4.81 \\
\hline ITL-12-07 × DVRT-2 & 8.67 & -16.13 & 0.00 & 3.74 & -8.41 & -9.30 & 4.07 & -5.13 & -12.29 & 183.67 & 1.29 & 9.76 & 4.49 & -15.56 & -10.27 & 0.82 & 18.66 & 19.23 \\
\hline ITL-12-07 × Punjab Chhuhara & 6.00 & $-37.93 * *$ & $-30.77 *$ & 3.96 & $-36.39 * *$ & -4.04 & 4.70 & 9.49 & 1.22 & 143.67 & $-20.77 * *$ & $-14.14 *$ & 5.12 & -11.62 & 2.40 & 0.61 & $-24.07 *$ & -12.02 \\
\hline JTL-16-03 × JT-3 & 8.33 & -10.71 & -3.85 & 4.11 & -0.32 & -0.32 & 4.56 & -1.51 & -1.51 & 168.67 & -5.77 & 0.80 & 5.65 & 12.93 & 12.93 & 0.64 & -8.17 & -8.17 \\
\hline TTL-16-03 $\times$ AT-3 & 7.67 & -17.86 & -11.54 & 4.92 & 8.29 & $19.32 * *$ & 4.84 & 5.21 & 4.53 & 174.33 & -2.61 & 4.18 & 4.78 & -4.40 & -4.40 & 0.76 & $-26.92 * *$ & 9.62 \\
\hline ITL-16-03 × DVRT-2 & 8.67 & -16.13 & 0.00 & 4.19 & 5.28 & 1.54 & 4.47 & 10.83 & -3.59 & 183.67 & 1.29 & 9.76 & 5.16 & 5.02 & 3.20 & 0.58 & -14.15 & -15.38 \\
\hline ITL-16-03 × Punjab Chhuhara & 6.33 & $-32.14 *$ & -26.92 & 4.10 & $-34.03 * *$ & -0.49 & 4.39 & $17.38^{*}$ & -5.32 & 143.67 & $-19.74 * *$ & $-14.14 *$ & 4.83 & -16.69 & -3.47 & 0.76 & -5.39 & 9.62 \\
\hline ITL-16-07 × JT-3 & 10.00 & 15.38 & 15.38 & 4.36 & 5.74 & 5.74 & 4.86 & 4.74 & 4.74 & 181.33 & 2.64 & 8.37 & 4.05 & $-30.71 * *$ & -19.07 & 0.67 & -18.70 & -3.85 \\
\hline ITL-16-07 $\times$ AT-3 & 10.00 & 11.11 & 15.38 & 4.20 & -7.63 & 1.78 & 5.11 & 10.93 & 10.21 & 176.67 & 0.00 & 5.58 & 4.07 & $-30.25 * *$ & -18.53 & 0.66 & $-36.86 * *$ & -5.29 \\
\hline ITL-16-07 × DVRT-2 & 8.67 & -16.13 & 0.00 & 4.10 & 3.19 & -0.49 & 5.14 & $27.36 * *$ & 10.78 & 169.67 & -6.43 & 1.39 & 4.61 & $-21.00 *$ & -7.73 & 0.68 & -17.07 & -1.92 \\
\hline ITL-16-07 × Punjab Chhuhara & 7.67 & -14.81 & -11.54 & 4.18 & $-32.85 * *$ & 1.29 & 4.39 & 11.14 & -5.32 & 161.33 & -8.68 & -3.59 & 5.16 & -11.64 & 3.20 & 0.72 & -11.79 & 4.33 \\
\hline ITL-15-02 $\times$ JT-3 & 8.33 & -3.85 & -3.85 & 3.28 & $-20.45 * *$ & $-20.45 * *$ & 4.40 & -5.10 & -5.10 & 166.33 & -0.60 & -0.60 & 4.59 & -13.35 & -8.27 & 0.78 & 12.50 & 12.50 \\
\hline ITL-15-02 × AT-3 & 8.33 & -7.41 & -3.85 & 4.13 & -9.02 & 0.24 & 3.99 & -13.46 & $-14.02 *$ & 176.67 & 2.71 & 5.58 & 4.33 & -18.14 & -13.33 & 0.67 & $-35.58 * *$ & -3.37 \\
\hline ITL-15-02 × DVRT-2 & 9.67 & -6.45 & 11.54 & 4.35 & 9.22 & 5.34 & 4.69 & 10.62 & 1.08 & 176.67 & -2.57 & 5.58 & 4.07 & $-23.05 *$ & -18.53 & 0.87 & $27.80 *$ & $25.96 *$ \\
\hline ITL-15-02 $\times$ Punjab Chhuhara & 9.33 & 3.70 & 7.69 & 4.21 & $-32.21 * *$ & 2.26 & 4.42 & 4.41 & -4.60 & 181.33 & 8.37 & 8.37 & 4.13 & $-28.65 * *$ & -17.33 & 0.74 & -7.88 & 6.73 \\
\hline ITL-12-02 $\times$ JT-3 & 8.00 & -7.69 & -7.69 & 4.36 & 5.66 & 5.66 & 4.72 & 1.87 & 1.87 & 174.33 & 4.18 & 4.18 & 5.53 & 10.53 & 10.53 & 0.67 & -2.40 & -2.40 \\
\hline ITL-12-02 $\times$ AT-3 & 8.00 & -11.11 & -7.69 & 4.20 & -7.63 & 1.78 & 4.75 & 3.11 & 2.44 & 174.33 & 1.36 & 4.18 & 4.15 & -17.07 & -17.07 & 0.66 & $-36.86 * *$ & -5.29 \\
\hline ITL-12-02 $\times$ DVRT-2 & 9.67 & -6.45 & 11.54 & 4.04 & 1.68 & -1.94 & 4.26 & -1.99 & -7.98 & 183.67 & 1.29 & 9.76 & 4.79 & -0.69 & -4.13 & 0.70 & 2.93 & 1.44 \\
\hline ITL-12-02 × Punjab Chhuhara & 7.67 & -14.81 & -11.54 & 4.69 & $-24.54 * *$ & $13.82 *$ & 4.81 & 10.57 & 3.81 & 174.33 & 4.18 & 4.18 & 4.35 & $-24.97 *$ & -13.07 & 0.67 & -16.18 & -2.88 \\
\hline ITL-16-05 $\times$ JT-3 & 8.33 & -3.85 & -3.85 & 4.33 & -1.29 & 5.01 & 4.49 & -3.09 & -3.09 & 186.00 & 11.16 & 11.16 & 3.81 & $-23.73 *$ & $-23.73 *$ & 0.93 & $35.10 * *$ & $35.10 * *$ \\
\hline ITL-16-05 × AT-3 & 8.00 & -11.11 & -7.69 & 4.00 & $-11.96 *$ & -2.99 & 4.42 & -3.98 & -4.60 & 183.67 & 6.78 & 9.76 & 4.12 & -17.60 & -17.60 & 0.75 & $-27.56 * *$ & 8.65 \\
\hline ITL-16-05 × DVRT-2 & 8.67 & -16.13 & 0.00 & 4.26 & -3.04 & 3.15 & 4.81 & 8.67 & 3.67 & 186.00 & 2.57 & 11.16 & 4.60 & -7.38 & -8.00 & 0.79 & 16.59 & 14.90 \\
\hline TTL-16-05 × Punjab Chhuhara & 9.67 & 7.41 & 11.54 & 4.39 & $-29.47 * *$ & 6.39 & 4.35 & -1.73 & -6.25 & 181.33 & 8.37 & 8.37 & 5.06 & -12.66 & 1.20 & 0.66 & -17.84 & -4.81 \\
\hline ITL-16-08 $\times$ JT-3 & 9.33 & 7.69 & 7.69 & 4.25 & -2.60 & 2.91 & 4.14 & -10.91 & -10.78 & 174.33 & 4.18 & 4.18 & 4.21 & -15.87 & -15.87 & 0.66 & -9.17 & -4.81 \\
\hline ITL-16-08 $\times$ AT-3 & 5.00 & $-44.44 * *$ & $-42.31 * *$ & 4.31 & -5.28 & 4.37 & 4.58 & -1.29 & -1.15 & 167.33 & -2.71 & 0.00 & 4.53 & -9.47 & -9.47 & 0.70 & $-33.01 * *$ & 0.48 \\
\hline ITL-16-08 $\times$ DVRT-2 & 5.67 & $-45.16 * *$ & $-34.62 *$ & 4.43 & 1.53 & 7.28 & 5.15 & 10.98 & 11.14 & 159.00 & $-12.32 *$ & -4.98 & 4.80 & 4.96 & -4.00 & 0.67 & -7.80 & -3.37 \\
\hline ITL-16-08 $\times$ Punjab Chhuhara & 5.67 & $-37.04 * *$ & $-34.62 *$ & 4.15 & $-33.33 * *$ & 0.57 & 4.05 & -12.92 & -12.80 & 146.33 & -12.55 & -12.55 & 5.13 & -11.51 & 2.53 & 0.71 & -11.62 & 2.40 \\
\hline ITL-17-06 $\times$ JT-3 & 8.33 & -3.85 & -3.85 & 4.40 & 1.69 & 6.71 & 4.49 & -3.16 & -3.16 & 181.33 & 8.37 & 8.37 & 5.05 & -1.49 & 1.07 & 0.72 & 3.37 & 3.37 \\
\hline ITL-17-06 $\times$ AT-3 & 8.00 & -11.11 & -7.69 & 4.07 & $-10.34 *$ & -1.21 & 4.52 & -1.88 & -2.52 & 170.67 & -0.78 & 1.99 & 5.54 & 7.99 & 10.80 & 0.53 & $-49.36 * *$ & $-24.04 *$ \\
\hline ITL-17-06 $\times$ DVRT-2 & 9.00 & -12.90 & 3.85 & 4.18 & -3.31 & 1.46 & 4.20 & -8.83 & -9.49 & 179.00 & -1.29 & 6.97 & 4.31 & -16.05 & -13.87 & 0.58 & -15.46 & -15.87 \\
\hline ITL-17-06 $\times$ Punjab Chhuhara & 10.00 & 11.11 & 15.38 & 3.89 & $-37.46 * *$ & -5.66 & 4.42 & -3.91 & -4.60 & 183.67 & 9.76 & 9.76 & 5.16 & -10.93 & 3.20 & 0.75 & -6.64 & 8.17 \\
\hline Mean & 8.21 & & & 4.21 & & & 4.48 & & & 172.64 & & & 4.85 & & & 0.707 & & \\
\hline C.D $(5 \%)$ & \multicolumn{3}{|l|}{2.47} & \multicolumn{3}{|l|}{0.47} & \multicolumn{3}{|l|}{0.60} & \multicolumn{3}{|l|}{23.63} & \multicolumn{3}{|l|}{1.08} & \multicolumn{3}{|l|}{0.20} \\
\hline C.V \% & \multicolumn{3}{|l|}{18.55} & \multicolumn{3}{|l|}{6.99} & \multicolumn{3}{|l|}{8.32} & \multicolumn{3}{|l|}{8.44} & \multicolumn{3}{|l|}{13.74} & \multicolumn{3}{|l|}{18.15} \\
\hline
\end{tabular}


Heterosis over standard check ranged from 42.31 per cent (JTL-16-08 $\times$ AT-3) to 23.08 per cent $(\mathrm{JTL}-15-05 \times \mathrm{JT}-3)$. Heterosis over better parent for fruit polar diameter $(\mathrm{cm})$ ranged from -37.46 per cent (JTL-17-06 $\times$ Punjab Chhuhara) to 9.22 per cent (JTL-15-02 $\times$ DVRT-2). Heterosis over standard check ranged from -20.45 per cent (JTL-15-02 $\times$ JT3) to 19.32 per cent (JTL-16-03 $\times$ AT-3). Significant positive heterosis has been also reported Droka et al., (2013), Yadav et al., (2013).Heterosis over better parent for fruit equatorial diameter $(\mathrm{cm})$ ranged from -13.46 per cent (JTL-15-02 $\times$ AT-3) to 27.36 per cent (JTL-16-07 $\times$ DVRT-2). Heterosis over standard check ranged from -14.02 per cent (JTL-15-02 $\times$ AT-3) to 11.14 per cent (JTL16-08 $\times$ DVRT-2). Significant positive has been also reported by Gul et al., (2010), Chattopadhyay and Paul (2012), Singh et al., (2012), Droka et al., (2013), Yadav et al., (2013), Sharma and Singh (2018).Heterosis over better parent for days to last harvest ranged from -20.77 per cent (JTL-12-07 $\times$ Punjab Chhuhara) to 11.16 per cent (JTL-16$05 \times$ JT-3). Heterosis over standard check ranged from -14.14 per cent (JTL-12-07 $\times$ Punjab Chhuhara and JTL-16-03 $\times$ Punjab Chhuhara) to 11.16 per cent (JTL-15-05 $\times$ JT3, JTL-16-05 $\times$ JT-3 and JTL-16-05 $\times$ DVRT2). Heterosis over better parent for total soluble solid ( ${ }^{\circ}$ Brix) ranged from -30.71 per cent $(\mathrm{JTL}-16-07 \times \mathrm{JT}-3)$ to 12.93 per cent (JTL-16-03 × JT-3). Heterosis over standard check ranged from -23.73 per cent (JTL-16$05 \times \mathrm{JT}-3)$ to 12.93 per cent $(\mathrm{JTL}-16-03 \times \mathrm{JT}-$ 3). Heterosis over better parent for acidity (\%) ranged from -49.36 per cent (JTL-17-06 $\times$ AT-3) to 35.10 per cent (JTL-16-05 $\times$ JT-3). Heterosis over standard check ranged from 24.04 per cent (JTL-17-06 $\times$ DVRT-2) to 35.10 per cent $($ JTL-16-05 $\times$ JT-3).Significant positive heterosis has been reported by Chattopadhyay and Paul (2012), Garg et al., (2013), Panchal et al., (2017), Sureshkumara et al., (2017), Sundharaiya et al., (2018).
In conclusion, the present investigation suggests that study resulted into identification of hybrid for tomato fruit yield \& its component traits. The cross JTL-15-02 $\times$ Punjab Chhuhara exhibited the highest desirable heterosis over better parent followed by JTL-15-05 × DVRT-2. These hybrids exhibited desirable heterosis for important yield attributes suggesting that the heterosis for marketable yield was associated with heterosis for component characters.

\section{References}

Adnan, R., Ahmed, S., Ghulam, M. W., Malik, M. S., Muhammad, A., Khanzada, H., Ayaz, A, K., Abdul, Q. and Ahmed, A. 2018. Estimation of hybrid vigor for yield and yield related traits in tomato (Solanum lycopersicon Mill.). Int. J. Bio. sci., 12(1):160-167.

Ahmed, S., Quamruzzaman, A. K. M. and Uddin, M. N. 2009. Combining ability estimates of tomato (Solanum lycopersicum L.) in late summer. SAARC J. of Agric., 7(1): 43-55.

Chattopadhyay, A. and Paul, A. 2012. Studies on Heterosis for yield and its Attributing Traits in Tomato (Solanum lycopersicum L.). Int. J. of Agric. Env. Biotech, 5(4): 405-410.

Droka, D., Kumar, R., Joshi, S. and Yadav, R. K. 2013. Genetic studies of quality traits in tomato (Solanum lycopersicum L.) under low temperature. Veg. Sci., 39(2): 189-191.

F.A.O., 2016. Area, production and productivity of horticultural CropsWorld. Available at http://www.fao.org/faostat/en accessed on $04^{\text {th }}$ December 2018.

Garg, N., Cheema, D. S. and Chawla, N. 2013. Manifestation of heterosis for fruit yield, quality and shelf-life in tomato (Solanum lycopersicum L.). Int. J. of Veg. Sci., 40(1): 28-33. 
Gautam, N., Kumar, M., Sandeep, K. and Sharma, S. 2018. Heterosis studies for yield and its components in tomato (Solanum lycopersicum L.) under North Western Himalayan Region, India. Int. J. Curr. Microbiol. App. Sci., 7(2): 1949-1957.

Hedrick, U. P. and Booth, N. O. 1908. Mendelian characters in tomatoes. Proceedings of theAmerican Soc. for Horti. Sci., 5(5): 19-24.

Indian Horticulture Database, 2018. Area, production and productivity of horticultural crops-all India. Available at http://www.indiastat.com accessed on $15^{\text {th }}$ May 2019.

Joshi, A., Thakur, M. C. and Kohli, U. K. 2005. Heterosis and combining ability for shelf life, whole fruit firmness and related traits in tomato. Ind. J. of Horti., 62(1): 33-36.

Kattegoudar, J., Lingaiah, H. B., Mamtha, N. C. and Arunkumar, B. 2018. Heterosis studies for yield and yield attributing characters of tomato (Solanum lycopersicum L.). Int. J. Curr. Microbiol. App. Sci., 7(1): 1073-1080.

Marbhal, S. K., Ranpise, S. A. and Kshirsagar, D. B. 2016. Heterosis study in cherry tomato for quantitative traits. Int. Res. J. Multid. Stud., 2(2): 1-6.

Panchal, B. B., Gurjar, T. D., Kalaria, V. D. and Patel, N. B. 2017. Study of heterosis breeding for processing characters in tomato (Solanum lycopersicum L.) Int. J. Pure App. Biosci., 5(4): 592-600.

Rana, A. and Vidyasagar, G. 2005. Exploitation of heterosis for yield and certain quality traits in tomato (Lycopersicon esculentum Mill.). J. of Horti. Sci., 11(2): 67-70.

Rattan, P. 2007. Line $\times$ Tester analysis involving bacterial wilt resistant genotypes across environments in tomato (Lycopersicon esculentum
Mill.). Ph. D. Thesis, p 248. Department of Vegetable Science and Floriculture, CSK Krishi Vishvavidyalaya, Palampur, Himachal Pradesh, India.

Sekhar, L., Prakash, B. G., Salimath, P. M., Channayya, H. P., Sridevi, O. and Patil, A. A. 2010. Implications of heterosis and combining ability among productive Single cross hybrids in tomato. Electr. J. Plant Breed., 1(4): 706-711.

Sharma, A. and Singh, J. P. 2018. Heterosis for fruit quality components in tomato (Solanum lycopersicum L.). Int. J. Curr. Microbiol. App. Sci., 6: 2827-2831.

Singh, N. B., Paul, A., Wani, S. H. and Kumar, M. L. 2012. Heterosis studies for yield and its components in tomato (Solanum lycopersicum L.). Int. J. of Life Sci., 1(3): 224-232.

Singh, P. K., Dasgupta, S. K. and Tripathi, S. K. 2004. Hybrid Vegetable Development, Food Product Press, Alice Street, Binghamton, New York. 6(4): 115.

Soni, N. K. and Soni, V. 2010. Fundamentals of Botany, $\left(1^{\mathrm{st}} E d\right.$.), Tata McGraw Hill Education Private Limited, New Delhi. pp. 333.

Sundharaiya, K., Karuthamani, M. and Sathish, G. 2018. Estimate of heterosis and per se performance of tomato $F_{1}$ hybrids for leaf curl virus resistance. Int. J. Curr. Microbiol. App. Sci.,6: 189196.

Sureshkumara, B., Lingaiah, H. B., Shivapriya, M. and Pavithra, H. B. 2017. Evaluation of tomato genotypes for growth, yield and quality attributes under eastern dry zone of Karnataka, India. Int. J. Curr. Microbiol. App. Sci,6(11): 1922-1930.

Tamata, S. and Singh, J. P. 2017. Heterosis in Tomato for Growth and Yield Traits. Int. J. of Veg. Sci., 24(2): 169-179.

Triveni, D. P., Saidaiah, K., Reddy, R. and 
Pandravada, S. R. 2017. Studies on heterosis for yield and yield contributing traits in tomato (Solanum lycopersicum L.). Int. J. Pure Appl. Bio. Sci., 5(4): 1677-1685.

Yadav, S. K., Singh, B. K., Baranwal, D. K. and Solankey, S. S. 2013. Genetic study of heterosis for yield and quality components in tomato (Solanum lycopersicum). African J. Agric. Res., 8(44): 5585-5591.

Yordanov, M. 1983. Heterosis in tomato. Theor. Appl. Genetics, 6: 189-219.

\section{How to cite this article:}

Ghadage, N.C., G.U. Kulkarni, H.R. Meta and Raju Shyadambi. 2019. Heterosis Studies for Fruit Yield and its Components in Tomato (Solanum lycopersicum L.). Int.J.Curr.Microbiol.App.Sci. 8(07): 1011-1020. doi: https://doi.org/10.20546/ijcmas.2019.807.121 